\title{
Hypophosphatemic osteomalacia: an unusual clinical presentation of multiple myeloma
}

\author{
M. Reyskens • K. Sleurs • L. Verresen • M. Janssen • \\ J. van den Berg $\cdot$ P. Geusens
}

Received: 27 November 2014 / Accepted: 25 February 2015 / Published online: 24 April 2015

(C) The Author(s) 2015. This article is published with open access at Springerlink.com

\begin{abstract}
An unusual case of a 75-year-old man is presented who had multiple stress fractures due to adult onset hypophosphatemic osteomalacia, which was the result of Fanconi syndrome, with light chain cast proximal tubulopathy due to multiple myeloma. A 75-year-old man presented with diffuse pain and muscle weakness. He had multiple stress fractures, low serum phosphate, decreased renal tubular reabsorption of phosphate, and normal PTH and FGF23, indicating adult onset hypophosphatemic osteomalacia. Phosphate supplements with calcitriol resulted in clinical recovery and healing of stress fractures. Because of proteinuria, a renal biopsy was performed that revealed Fanconi syndrome with light chain cast proximal tubulopathy and light kappa chains were found in serum and urine. A bone biopsy confirmed the diagnosis of multiple myeloma, and treatment with chemotherapy resulted in cytological and clinical recovery.
\end{abstract}

\author{
M. Reyskens $\cdot$ K. Sleurs $\cdot$ L. Verresen \\ University Hasselt, Diepenbeek, Belgium \\ M. Reyskens \\ e-mail: margotreyskens@gmail.com \\ K. Sleurs \\ e-mail: kristiensleurs@hotmail.com \\ L. Verresen \\ e-mail: Luc.Verresen@zol.be \\ L. Verresen $\cdot$ M. Janssen \\ ZOL, Genk, Belgium \\ M. Janssen \\ e-mail: mia.janssen@zol.be \\ M. Janssen \\ Limburg Oncologic Center, Hasselt, Belgium \\ J. van den Berg \\ Department of Internal Medicine, VieCuri Medical Centre, PO Box \\ 1926, 5900 BX Venlo, The Netherlands \\ e-mail: j@vdbergh.org
}

Keywords Fanconi syndrome · Multiple myeloma · Myeloma cast nephropathy $\cdot$ Osteomalacia $\cdot$ Stress fracture

\section{Introduction}

Diffuse pain is a common complaint of patients in rheumatologic practice. It is associated with a variety of diseases, such as fibromyalgia, thyroid disease, body-wide infection, overuse, polymyalgia rheumatica, multiple myeloma, and metabolic bone diseases, such as Paget's disease, hyperparathyroidism, and osteomalacia [1]. Medical history, clinical examination, laboratory examinations, and radiography enable to make the diagnosis of the most common conditions.

\section{J. van den Berg}

Department of Internal Medicine, NUTRIM School for Nutrition, Toxicology and Metabolism, Maastricht University Medical Centre (MUMC), PO Box 616, 6200 MD Maastricht, The Netherlands

J. van den Berg $\cdot$ P. Geusens

Biomedical Research Centre, Hasselt University,

Agoralaan - gebouw D, 3590 Diepenbeek, Belgium

\section{P. Geusens}

Department of Internal Medicine, Subdivision Rheumatology, CAPHRI, Maastricht University Medical Centre (MUMC), PO Box 616, 6200 MD Maastricht, The Netherlands

P. Geusens $(\bowtie)$

Department of Medicine, Subdivision of Rheumatology,

Academic Hospital azM, P. Debyelaan 25, Postbus 5800, 6202 AZ Maastricht, The Netherlands

e-mail: piet.geusens@scarlet.be 
Unexplained diffuse pain together with increased bonespecific alkaline phosphatases needs more specific imaging, such as bone scintigraphy [1]. Paget's disease is characterized by typical mono- or poly-osteal involvement. Osteoblastic metastases show multiple and diffuse focal lesions, while osteolytic lesions cannot be identified. Osteomalacia is characterized by focal increased uptake at stress locations, such as around the joints, in the vertebrae, and in the ribs.

Osteomalacia in adults is characterized by bone pain and muscle weakness. The most frequent cause is vitamin D deficiency in the elderly. Other causes include malabsorption (celiac disease, Crohn's disease, gastrectomy, gastric bypass, bowel resection, pancreatitis), impaired hydroxylation of vitamin D substrate (severe chronic liver disease, renal osteodystrophy), anti-convulsant therapy, and hypophosphatemic osteomalacia due to phosphate wasting (oncogenic osteomalacia and Fanconi syndrome) [1].

Diffuse bone pain is the most frequent symptom of multiple myeloma (MM), reported in more than half of patients [2]. Myeloma plasma cells produce receptor activator of the nuclear factor kappa B (RANKL), IL-6 and macrophage inflammatory protein- $1 \alpha$ (MIP1 $\alpha)$, that increase bone resorption, Dickkopf 1 (DKK1), IL-3, IL-7, hepatic growth factor (HGF), soluble frizzled receptor-like proteins (sFRP) and sclerostin that decrease bone formation, and TNF $\alpha$ and Activin A that have mixed effects on bone cells, resulting in increased vertebral fracture risk in patients with monoclonal gammopathy of undetermined significance (MGUS) and nonhealing osteolytic lesions with increased risk of pathological fractures [3-8] (Fig. 1). Renal impairment is found in 20-
$40 \%$ of patients, mainly as a result of direct tubular damage from excess protein load, dehydration, hypercalcemia, and the use of nephrotoxic medications [8].

In contrast, diffuse bone pain in association with acquired Fanconi syndrome leading to hypophosphatemic osteomalacia is only rarely reported in association with $\operatorname{MM}[9,10]$. The pathophysiology of this bone disease is totally different from the classical osteolytic lesions. Here, monoclonal kappa light chain paraproteins precipitate as crystal casts in the proximal renal tubule by binding to Tamm-Horsfall glycoproteins (THP), causing Fanconi syndrome with renal phosphate wasting. This results in hypophosphatemia with osteomalacia with stress fractures (Looser zones) without osteolytic lesions, and with decreased energy metabolism resulting in muscle weakness. The clinical course is distinct and characterized a slow progressive course with an early phase dominated by metabolic complications [10].

\section{Case summary}

A 75-year-old man was referred because of chronic back pain with increased alkaline phosphatase. The pain was localized in the vertebral column and radiated to the chest. Physical examination showed a dorsal hyperkyphosis and restriction of spine movement. Laboratory results were as follows (normal ranges in parentheses): serum creatinine $1.55 \mathrm{mg} / \mathrm{dL}(0.50-1.20)$, eGFR $44 \mathrm{~mL} / \mathrm{min} / 1.73 \mathrm{~m}^{2}(>60)$, uric acid $1.0 \mathrm{mg} / \mathrm{dL}(3.4$ 7.0), alkaline phosphatase $891 \mathrm{U} / \mathrm{L}$ (40-130), serum phosphorus $0.46 \mathrm{mmol} / \mathrm{L}(0.74-1.19)$, and normal protein
Fig. 1 Schematic representation of pathophysiologic mechanisms of bone involvement in MM. THP Tamm-Horsfall glycoproteins, $P T C$ proximal tubular cells, Glom glomerulus, $R A N K L$ receptor activator of the nuclear factor kappa B, DKK1 dickkopf1, $\mathrm{Ocl}$ osteoclast, $\mathrm{Obl}$ osteoblast, MIP $1 \alpha$ macrophage inflammatory protein- $1 \alpha, H G F$ hepatic growth factor, $s F R P$ soluble frizzled receptor-like proteins, $A K I$ acute kidney injury, $M G S S$ monoclonal gammopathy of skeletal significance

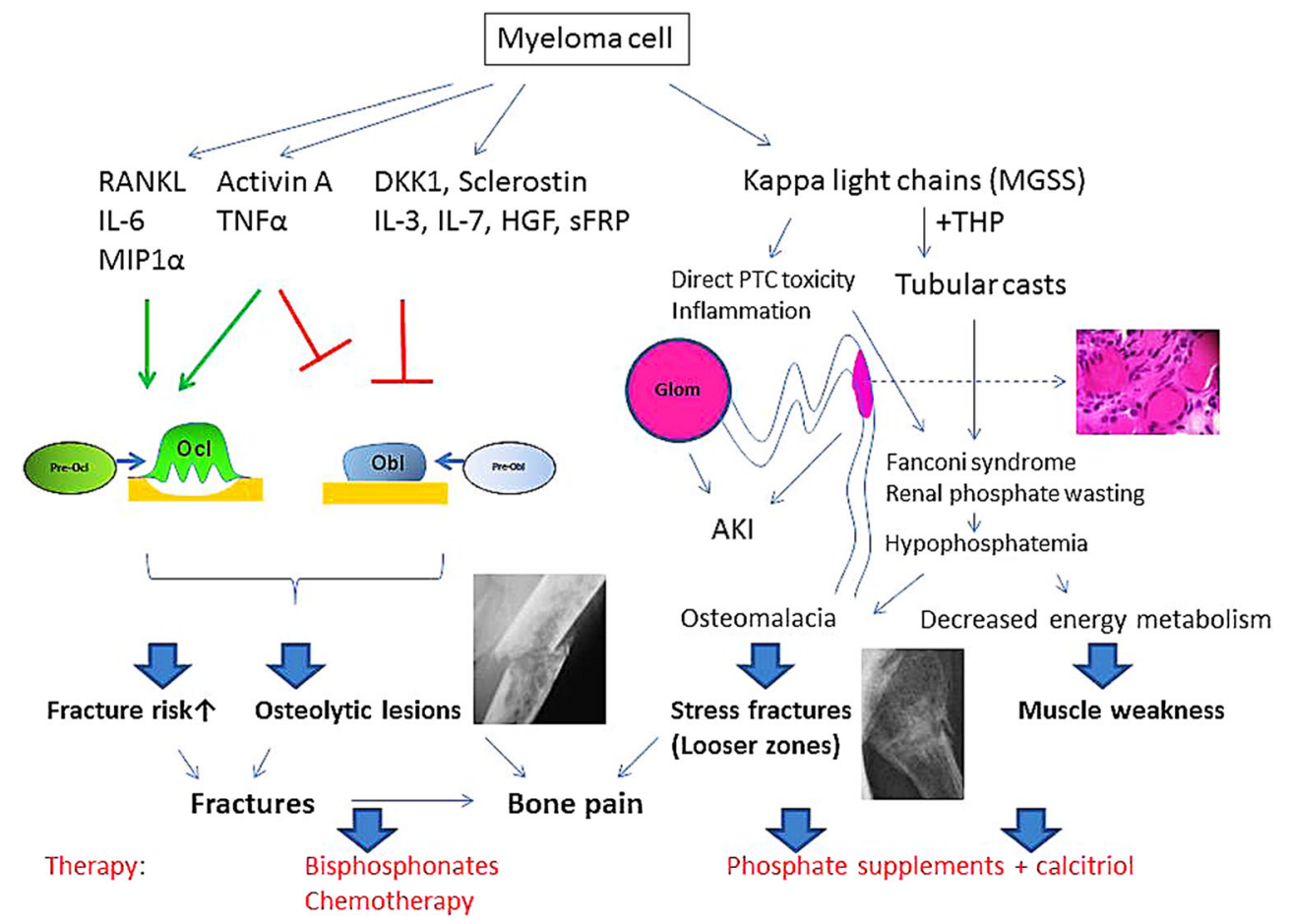


electrophoresis. CT of the thoracic wall and lumbar spine showed partially healed fractures of rib 7, 8, 9, and 10 and an old fracture of the processus transversus L3. MRI of the pelvis showed aspecific bone remodeling and bone edema in the right sacrum. The tentative diagnosis of Paget's disease was made and zoledronate $5 \mathrm{mg}$ IV with calcium and vitamin D supplements was given.

Because of increasing diffuse pains and difficulties of movement, the patient was referred for a second opinion. He had extreme muscle weakness in shoulders and hips, and could barely stand up from chair and undress. Laboratory revealed increased alkaline phosphatase of $171 \mathrm{U} / \mathrm{L}$ (46\% bone fraction), low phosphate (0.40) and eGFR (47), and normal calcium, PTH, 25(OH)2D3, prostate specific antigen, protein electrophoresis, and TSH. Bone scintigraphy showed multiple uptake zones in ribs, vertebrae (D6, 7, 8, and 10), right femoral neck, left acetabulum, left patella, right proximal tibial plateau, proximal left fibula, right distal tibia, and left calcaneus. Radiographs showed multiple stress fractures at these sites, but no osteolytic zones. MRI of the spine indicated vertebral fractures (D7, 8, and 10) without osteolytic zones. Bone mineral density was low, with a T-score of -3.6 in the spine and -2.5 in the total hip. The diagnosis of adult-onset hypophosphatemic osteomalacia was made. Further examinations indicated decreased tubular reabsorption of phosphate (49\%), a positron emission tomography (PET) revealed no tumors or active granulomatous disease, and normal serum value of FGF-23, excluding tumor-induced osteomalacia syndrome (TIO) as a cause. Treatment with phosphate, calcitriol, and calcium supplements resulted in spectacular clinical amelioration of muscle weakness, normalization of serum phosphate and alkaline phosphatase, radiographic healing of the stress fractures within 6 months, and an increase in BMD of $30 \%$ in the spine and of $9 \%$ in the total hip after 18 months (T-score of -1.5 and -2.0 , respectively).

Because of the deteriorating renal function $(\mathrm{eGFR}=30)$ and no TIO was found, urine analysis was performed showing proteinuria and glucosuria, and the patient was referred to a nephrologist. A mixed proteinuria $(3 \mathrm{~g} / \mathrm{d})$ with excretion of type kappa monoclonal light chains was found, together with hypouricemia $(1.23 \mathrm{mg} / \mathrm{dl})$, renal glycosuria $(696 \mathrm{mg} / \mathrm{dl})$, and proximal tubular acidosis (urine $\mathrm{pH} 7$ and serum bicarbonate $18.3 \mathrm{meq} / \mathrm{l}$ ), indicating damage of the proximal tubular cells, compatible with Fanconi syndrome. An excess of kappa light chains was also detected in blood with at a concentration of $401 \mathrm{mg} / \mathrm{l}$. A renal biopsy showed normal glomeruli and intratubular eosinophilic casts and damaged tubular epithelial cells, suggestive for myeloma cast nephropathy. The diagnosis of MM was confirmed by the oncologist on a bone marrow biopsy and treatment with 8 cycles of bortezomib and dexamethasone was started. Treatment with phosphate, calcium, and vitamin D supplements was also continued. Five months after starting chemotherapy, with three cycles of bortezomib and dexamethasone, the renal function was stabilized (eGFR= 43). A control bone biopsy with immunophenotyping showed disappearance of monoclonal plasma cells in the bone marrow. However, kappa light chains were still present in serum $(102 \mathrm{mg} / \mathrm{l})$ and urine $(150 \mathrm{mg} / \mathrm{l})$, with persistent proteinuria with tubular proteins and TRP was still low (0.61). Although the patient is now clinically fully recovered without pain and without complaints of muscle weakness, he still has to take calcitriol and phosphate supplements, and is further in follow up by the oncologist.

\section{Discussion}

We present a case of sporadic adult-onset hypophosphatemic osteomalacia due to Fanconi syndrome secondary to light chain kappa deposition by MM. This is an exceptional case of $\mathrm{MM}$ as diffuse bone pains due to osteolytic lesions and secondary fractures are the most frequent and typical clinical manifestation of bone involvement. But in this case, bone pain was the result of osteomalacia with stress fractures without osteolytic lesions and accompanied by extreme muscle weakness.

The clinical challenge to diagnose MM in this patient was that the initial complaints and laboratory findings directed to the diagnosis of osteomalacia, and that the clinical symptoms disappeared and stress fractures healed with phosphate supplements and calcitriol. Interestingly, phosphate and calcitriol treatment resulted in a major increase of BMD in the spine and hip, indicating a generalized major correctible mineralization deficit due to osteomalacia. Only later, the diagnosis of Fanconi syndrome with MM cast nephropathy lead to the diagnosis of MM.

Causes of adult onset hypophosphatemic osteomalacia (AOHO) include increased urinary loss by decreased renal tubular reabsorption, impaired intestinal absorption, and shifts of extracellular phosphate into cells [1]. Chronic hypophosphatemia usually signifies a persistent renal tubular phosphate-wasting disorder, which can be the result of PTH/ PTHrP-dependent causes (such as hyperparathyroidism) and PTH/PTHrP-independent causes. This last group includes excess of FGF23 (such as TIO), intrinsic renal diseases (such as Fanconi syndrome), other systemic disorders, and drugs/ toxins. Autosomal dominant hypophosphatemic rickets (ADHR) only rarely presents in adulthood [11]. In this patient, the diagnosis of $\mathrm{AOHO}$ was appropriate, and treatment with phosphate supplements resulted in clinical recovery of bone pain and muscle weakness and radiographic healing of the stress fractures. In this patient, TIO could be excluded (normal total body PET scan, normal FGF23). Because of decreased renal function, urine analysis was 
performed, indicating proteinuria, and Fanconi syndrome was diagnosed.

In patients with Fanconi syndrome, 15/32 presented with bone pain [9]. Rao reported 2 patients with Fanconi syndrome, one of whom the initial complaint was muscle weakness [11]. Proximal tubulopathy is the result of monoclonal light chains that precipitate with Tamm-Horsfall glycoproteins and casts are formed in the renal tubules. This results in inflammation and damaging of the tubules, followed by renal insufficiency if the damage is severe enough [12]. Besides cast nephropathy, free light chains also cause isolated proximal tubule cell cytotoxicity by activation of inflammatory cascades and cause acute kidney insufficiency [10]. Renal biopsy confirmed casting and light chains were demonstrated in blood and urine.

The clinical lesson from this patient is that bone disease in MM is heterogeneous (Fig. 1) and that in sporadic adult $\mathrm{AOHO}$ due to renal phosphate wasting presenting with bone pain and muscle weakness, and without TIO, the urine should be controlled for proteinuria and light chains should be searched in blood and urine. This case is also a nice example of the concept of monoclonal gammopathy of skeletal significance (MGSS) [13].

In conclusion, this case report indicates that in adult-onset sporadic osteomalacia, MM should be part of the differential diagnosis, when no TIO is detected, even when clinical recovery of bone pain and muscle weakness is achieved with phosphate and calcitriol supplements.

\section{Conflicts of interest None.}

Open Access This article is distributed under the terms of the Creative Commons Attribution Noncommercial License which permits any noncommercial use, distribution, and reproduction in any medium, provided the original author(s) and the source are credited.

\section{References}

1. Rosen C (2013) Primer on the metabolic bone diseases and disorders of mineral metabolism. John Wiley \& Sons, Iowa

2. Kyle RA, Rajkumar SV (2004) Multiple myeloma. N Engl J Med $351: 1860-1873$

3. Roodman GD (2002) Role of the bone marrow microenvironment in multiple myeloma. J Bone Miner Res 17:1921-1925

4. Glass DA 2nd, Patel MS, Karsenty G (2003) A new insight into the formation of osteolytic lesions in multiple myeloma. N Engl J Med 349:2479-2480

5. Melton LJ 3rd, Rajkumar SV, Khosla S, Achenbach SJ, Oberg AL, Kyle RA (2004) Fracture risk in monoclonal gammopathy of undetermined significance. J Bone Miner Res 19:25-30

6. Melton LJ 3rd, Kyle RA, Achenbach SJ, Oberg AL, Rajkumar SV (2005) Fracture risk with multiple myeloma: a population-based study. J Bone Miner Res 20:487-493

7. Edwards CM, Zhuang J, Mundy GR (2008) The pathogenesis of the bone disease of multiple myeloma. Bone 42:1007-1013

8. Palumbo A, Anderson K (2011) Multiple myeloma. N Engl J Med 364:1046-1060

9. Ma CX, Lacy MQ, Rompala JF, Dispenzieri A, Rajkumar SV, Greipp PR et al (2004) Acquired Fanconi syndrome is an indolent disorder in the absence of overt multiple myeloma. Blood 104:40-42

10. Bhutani M, Klein MJ, Glezerman I, Landau H, Rosenzweig M, Hassoun H (2011) Osteomalacia due to adult Fanconi syndrome in multiple myeloma. Leuk Lymphoma 52:536-538

11. Rao DS, Parfitt AM, Villanueva AR, Dorman PJ, Kleerekoper M (1987) Hypophosphatemic osteomalacia and adult Fanconi syndrome due to light-chain nephropathy. Another form of oncogenous osteomalacia. Am J Med 82:333-338

12. Hutchison CA, Batuman V, Behrens J, Bridoux F, Sirac C, Dispenzieri A, International Kidney and Monoclonal Gammopathy Research Group et al (2011) The pathogenesis and diagnosis of acute kidney injury in multiple myeloma. Nat Rev Nephrol 8:43-51

13. Drake MT (2014) Unveiling skeletal fragility in patients diagnosed with MGUS: no longer a condition of undetermined significance? J Bone Miner Res 29(12):2529-2533 